\title{
THE GSI-LBL PLASTIC BALL/WALL SPECTROMETER*
}

\author{
H.-P. GUSTAFSSON, K.G.R. DOSS, H.H. GUTBROD, B. KOLB, \\ B. LUDEHIGT, A.M. POSKANZER, T. RENNER, H. RIEDESEL, \\ H.G. RITTER, A. WARWICK AND H. WIEMAN \\ Gesellschaft fur Schwerionenforschung \\ D-6100 Darmstadt, West Germany
}

and

Nuclear Science Dirision

Lawrence Berkeley Laboratory

University of California

Berkeley, California 94720

For the Conference on Instrumentation for Heavy Ion Nuclear Research, Oak Ridge National Laboratoiry

\section{DISCLAIMER}

\begin{abstract}
This report was prepared as an account of work sponsored by an agency of the United States Government. Neither the United States Government nor any agency thereof, nor any of their employees, makes any warranty, express or implied, or sssumes any legal liability ur responsibility for the aceuracy, completeness, of usefulness of any information, apparatus, product, or process disclosed, or represents that its use would not in fringe privately owned rights. Reference herein to any specific co:nmereial product, process, or service by trade name, trademark, manufacturer, or otherwise does not necessarily constitute or imply its endorsement, recommendation, or favoring by the United States Government or any agency thereof. The views and opinions of authors expressed hersin do not necessarily state or reflect those of the United States Government or any agency thereof.
\end{abstract}

*This work was supported by the Director, Office of Energy Research, Division of Nuclear Physics of the Office of High Energy and Nuclear Physics of the U.S. Department of Eneroy under Contract DE-ACO3-76SF0009.9. 
THE GSI-LBL PLASTIC BALL/HALL SPECTROMETER*

H. -A. GUSTAFSSON, K.G.R. DOSS, H.H. GUTBROD, B. KOLB, B. LUDEWIGT, A.M. POSKANZER, T. RENNER, H. RIEDESEL, H.G. RITTER, A. WARWICK AND H. WIEMAN Gesellschaft fur Schwerionenforschung D-6100 Darmstadt, West Germany

and

Nuclear Science Division

Lawrence Berkeley Laboratory.

University of California

Berkeley, California 94720

For the high multiplicity events occurring in relativistic nuclear collisions an electronic $4 \pi$ detector with perticle identification has been built. I It consists of $815 \Delta E-E$ telescopes and 176 TOF telescopes covering 97 per cent of $4 \pi$. Very good particle identification has been obtained for hydrogen and helium isotopes and also $\pi^{+}$have been detected with high efficiency. This spectrometer was used at the Berkeley Bevalac to study the reactions $\mathrm{Ca}+\mathrm{Ca}, \mathrm{Nb}+\mathrm{Nb}$ and $\mathrm{Au}+\mathrm{Au}$ at energies between 0.15 and $1.05 \mathrm{GeV} /$ nucleon. Varjous exclusive observables like the degree of thermalization, the multiplicity dependence of composite particle formation, and freeze-out densities are discussed. From global analyses the collective flow of nuclear matter has been diduced. A method of separatirg efficientiy participant and spectator paricicles has been

*This work was supported by the Director, Office of Energy Research, Division of Nuclear Physics of the Office of High Energy and Nuclear Physics of the U.S. Department of Eitergy under Contract DE-ACO3-76SF00098. 


\section{H.-A. GUSTAFSSON, et al.}

applied in order to disentangle the two collective effects: The "side-splash" of the participants and the "bounce-off" in the fragmentation region.

\section{INTROOUCTION}

In relativistic nuclear collisions measurement of the charged particle multiplicity of an event has been founo to be an important characteristic of the underlying reaction mechanism. ${ }^{2}$ Further progress towards full event analysis is made very difficult because of the large dynamic range in fragment energy, fragment mass, and fragment multiplicity. In emulsion and streamer chamber studies, attempts have been made to fully reconstruct the events, but limits have had to be accepted in the quality of particle identification and total number of events analyzed. A detector system that can favorably complement 4n detectors like emulsions, AgCl detectors, or streamer chambers, has to have the capability of detecting and identifying as many charged particles as possible in sigh multiplicity events. Therefore, the concept of a $4 \pi$ detector consisting of many individual $\Delta E-E$ and time-of-flight telescopes was chosen, that promises fast data analysis.

For the coverage of most $4 \pi$, the Plastic Ball was built based on the geometry of the Crystal Ball at Stanford (Fig. 1). It completely surrounds the target, except for the extreme forward and backward angles. The Plastic Ball consists of $815 \Delta E$ - E particle identifying detector modules where the $\Delta E$ counter is a $C_{a}{ }_{2}(E u)$ crystal and the $E$ counter is a plastic scintillator. They are optically coupled and read out by one 


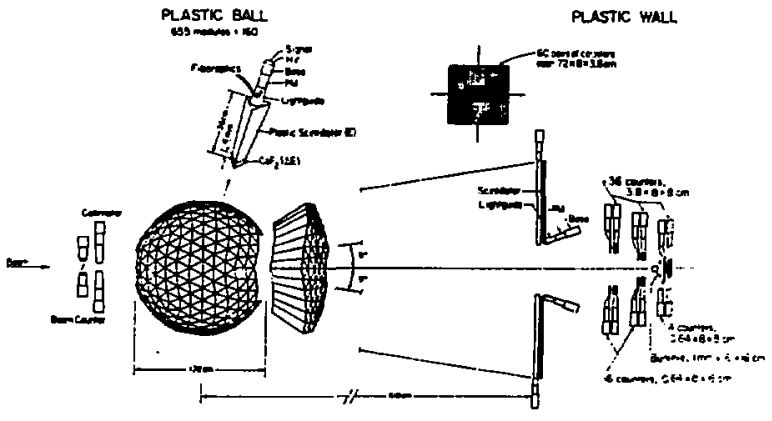

FIGURE 1 Schematic view of the Plastic Ball and the Plastic Wall

photomultiplier with subsequent separation of the signals by pulse shape analysis. This design allows one to cover a solid angle of up to 97 per cent of $4 \pi$.

The most forward angles ( 0 deg. - 10 deg.) are covered with a multi-element time-of-flight system (the Piastic Wall). The inner part of the Wall, covering the angular region from $0 \mathrm{deg}$. to $2.5 \mathrm{deg}$. , and the outer part, from $2.5 \mathrm{deg}$. to approximately $10 \mathrm{deg}$. The Plastic Wall serves in addition as a trigger counter for the Ball.

During many years of studying mostly inclusive observables in heavy ion collisions at relativictic 
H.-A. GUSTAFSSON, et al.

energies with light or medium heavy projectiles a wealth of information on nuclear fragmentation, particle production, and statistical properties of hot and dense nuclear matter has been accumulated and the complexity of the systems under investigation has been more and more uncovered. Various models based on rather different assumptions like the intranuclear cascade or the hydrodynamical model are able to reproduce the inclusive cross sections. Therefore the call for decisive experiments using exclusive observables became inevitable in orcer to draw a consistent picture of the behaviour of nuclear matter under extrene conditions and to gain information on the energy per nucleon as a function of density and temperature, i.e. the nuclear equation of state, from which the properties of nuclear systems created in energetic heavy ion collisions may consistently be derived. First attempts to relate the measured pion multiplicity to the equation of state have been made recentiy. ${ }^{3}$ Since heavy beams up to uranium are now available at the Bevalac, the total mass in the reaction volume is as close to nuclear matter as possible in the laboratory. Data from $4 \pi$ detectors like the plastic Ball are ideally suited to study the emission patterns and event shapes of high energy nuclear collisions which might be able to distinguish between predictions of cascade and hydrodynamical models.

\section{THE PLASTIC BALL}

2.1. General Characteristics

Each of the 815 detector modules represents a particle identifying telescope with a $\Delta E$ and $E$ detector using a 
THE GSI-LBL. PLASTIC BALL/WALL. SPECTROMETER

slow and a fast scintillator read out via one photomultiplier (Fig. 2). The $\Delta E$ counter is a $\mathrm{CaF}_{2}$ (Eu)

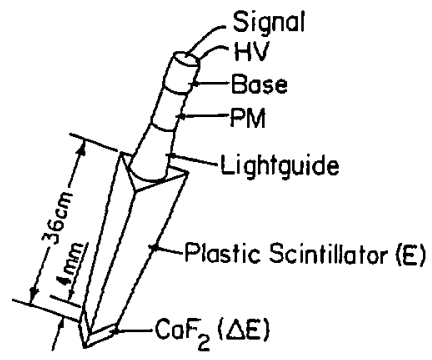

FIGURE 2 Schematic view of a single Plastic Ball module. crystal with a characteristic decay time of $1 \mu \mathrm{s}$ for the emission of the scintillation light. The $\Delta E$ light is read out through the $E$ counter (i.e., the $E$ counter serves as a light guide to the $\Delta E$ counter), which consists of a plastic scintillator. The $E$ signal is collected within $10 \mathrm{~ns}$.

The thickness of the $\triangle E \mathrm{CaF}_{2}(E u)$ crystal was chosen to be $4 \mathrm{~mm}$ with $35.6 \mathrm{~cm}$ as the length of the $E$ plastic scintillator. This allowed good $\Delta E$ signals even for minimum ionizing particles and also a minimum low energy cutoff for particles stopping in the $\Delta E$ counter. In order to obtain clean proton spectra up to $200 \mathrm{MeV}$, the detector length was chosen to stop $240 \mathrm{MeV}$ protons. This additional length assures that the punch through deuterons do not disturb tne proton spectra below 200 Mev. 
H.-A. GUSTAFSSON, et al.

\subsection{Pion Identification}

In designing the Plastic Ball, special care was taken to detect the positive pions. As the yield of the $\pi^{+}$is only about 10 per cent of the proton yield at beam energies well above the pion production threshold, in a pure $\Delta E$ - $E$ identification scheme the pions would be overshadowed by the background produced by heavier particles. Therefore, the $\pi^{+}$are additionally identified by their delayed decay. Stopped $\pi^{+}$decay into $a_{\mu}^{+}$and a neutrino with a mean life of $26 \mathrm{~ns}$. The $\mu^{+}$subsequently decays into a positron and two neutrinos with a mean life of $2.2 \mu \mathrm{s}$.

\section{THE PLASTIC WALL}

The Plastic Wall covers an area of $192 \mathrm{~cm} \times 192 \mathrm{~cm}$ and provides fine position resolution coverage of the angular region between $\theta=0$ deg. and the forwardmost sections of the Plastic Ball ( $\theta-10$ deg.). .

The outer Wall serves to extend the acceptance of the Ball-Wall system from $\theta=10 \mathrm{deg}$. to $\theta=2.5 \mathrm{deg}$. detecting particles in the same range of mass and charge as the Ball itself, but measuring velocity and $Z$ instead of energy, $Z$ and $A$ as in the Ball. The inner Wall extends the angular measurement to 0 deg. In addition, the center region of the inner Wall makes up the event trigger in the first round of experiments.

\subsection{The Outer Wall}

The outer Wall region consists of 60 pairs of counters, each $72 \times 8 \times 3.8 \mathrm{~cm}^{3}$, providing coincidence units sensitive to charged particles passing through both bars 


\section{THE GSI-LBL PLASTIC BALL/WALL SPECTROMETER}

of a pair. Neutron and gamma background is rejected by this coincidence requirement.

The coordinates of the point of incidence of the charged particle on the outer Wall are determined to within the width of the module $(8 \mathrm{~cm})$. in one direction and measured to the same accuracy along the length of the pair by measuring the light travel time to opposite ends of each of the two scintillators. A time resolution of 350 ps (fwhm) was achieved for minimum ionizing protons. The mean time of the pair gives the flight time from the target and hence the particle velocity.

\subsection{The Inner Wall}

The inner Wall covered the region within 2 deg. of the beam and was finely oivided because of the high multiplicity of fragments near the beam. Its main purpose was to form the fast trigger with the upstream beam counter and collimator to decide which events were to be recorded. of course, it aiso gave nuclear charge and velocity information for the particles it cietected and could be used for centering the beam on the Wall.

The inner Wall consisted of a $6 \times 6$ array of 36 thick scintillators $8 \times 8 \mathrm{~cm}^{2}$ each, $3.8 \mathrm{~cm}$ thick. In front of each was a $0.64 \mathrm{~cm}$ thin scintillator. For the center four counters the thin and thick scintillators were of the same area and in one to one correspondence, but for the remaining 32 thick scintillators, only 16 thin scintillators were used, each $8 \times 16 \mathrm{~cm}^{2}$ in area and covering a pair of thick detectors. In addition, in front of the center four pairs of scintillators was placed one $16 \times 16 \mathrm{~cm}^{2}$ "Bull's Eye" scintillator, oniy 
H.-A. GUSTAFSSON, et al.

$1 \mathrm{~mm}$ thick. The purpose of the bull's eye was to reject beam particles, and it was made thin to minimize nuclear interactions in the detector itself.

\section{PERFORMANCE}

\subsection{The Plastic Ball}

The tests performed at LAMPF low energy pion beam line and at the Berkeley 184" cyclotron with $800 \mathrm{MeV}$ a-particles showed that the energy resolution of a

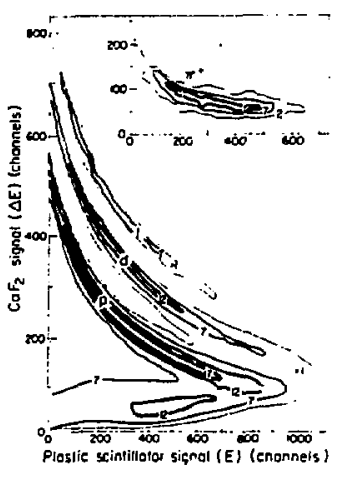

FIGURE $3 \Delta E-E$ contour diagram of 655 modules after gain matching and scattering out reconstruction of approximately one million reaction products. The contour lines are labeled as to their relative height in arbitrary units. The upper contour diagram for $\pi^{+}$is obtained by requiring a delayed decay signal measured in the 10 us TDCs. 
THE GSI-LBL PLASTIC BALL/HALL SPECTROMETER single module is sufficient to achieve the desired particle identification.

The first Bevalac experiment was performed in June 1981 with an $800 \mathrm{MeV} /$ nuclean Ne beam on a Pb target and ${ }^{40} \mathrm{Ca}$ beams at $400 \mathrm{MeV} /$ nucleon and $1.05 \mathrm{GeV} /$ nucleon on a calcium target. The quality of the particle identification for the 655 mcdules between $30 \mathrm{deg}$. and 160 deg. is shown in Fig. 3. A cut in the $\triangle E-E$ plane perpendicular to the particle identification lines is selected with $\Delta E$ and $E$ projected on this cut in a certain energy range (Fig. 4). The dashed curve

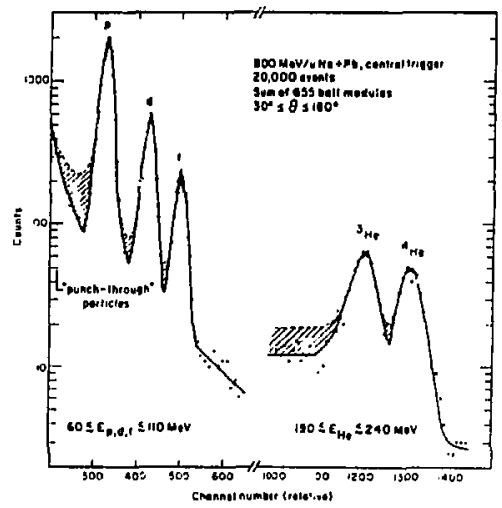

FIGURE 4 Particle identification spectrum for 655 modules after gain-matching and with and without scattering out reconstruction.

represents the raw data after gain matching, whereas the solid line shows the particle separation after all 


\section{H. -A. GUSTAFSSON, et al.}

scattered out particles have been reconstructed (Fig. 4). The effectiveness of the reconstruction has been shown before. 4

\subsection{The Plastic Wall}

Since the Plastic Wall can detect beam velocity particles the time calibration is taken from each individual experiment. In the $800 \mathrm{MeV} / \mathrm{nucleon}^{20} \mathrm{Ne}$ on $\mathrm{Pb}$ experiment a time resolution of $\sim 350$ ps was observed detween the inner Wall detectors and the beam counter. For the outer Wa11, where dominantly hydrogen and helium fragments are detacted, a pulse height resolution of $\sim 20-25$ per cent was good for an excellent sedaration of hydrogen, helium, and lithium.

These performance figures of the Ball and Wall allow one to draw a figure for the system's resporise as a function of fragment momentum and rapidity. Fig. 5 shows the response of the Ball and the Wall for protons or a-particles.

\section{RESULTS}

\section{I Stopping and Thermalization}

By measuring in each event the momentum distribution of the fragments in the center of mass system the degree of thermalization can be determined. For an isotropically expanding systern one finds in the center-of-mass frame from simple phase space considerations:

$$
\frac{2}{\pi} \sum_{i}\left|p_{1}^{i}\right|=\sum_{i}\left|p_{\|}^{i}\right|
$$

The sums in eq. (1) contain the perpendicular, $p_{1}$, and 
THE GSI-LBL PLASTIC BALL/WALL SPECTROMETER

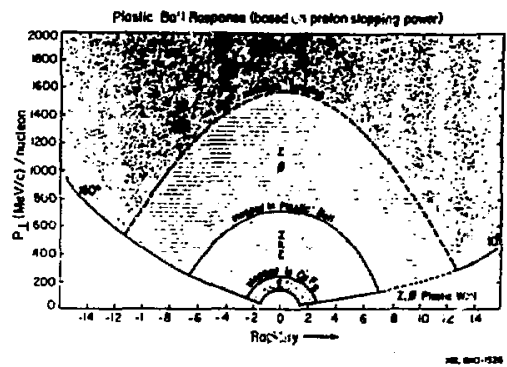

FIGURE 5 Plastic Ball and Plastic Wall response in the transverse momentum $p$ versus rapidity $y$ plane. The limits labeled "stopped in $\mathrm{CaF}_{2}$ " and "stopped in Plastic Ball" refer to protons and ${ }^{4} \mathrm{He}$ fragments.

longitudinal, $P_{\|}$, momentum components of all particles in an event. A global stopping of the two nuclei in the center of mass system would show up by the two sides of eq. (1) being equal, or $p_{\perp}$ even larger if flow into the transverse direction exists. In the presence of transparency $p_{\|}$would always be larger. Isotropy is a necessary but not sufficient condition for thermalization. If in addition the energy distributions are of Maxwel1Boltzmann type the emitting system may be called thermalized.

Plastic Ball data ${ }^{5}$ are shown in Fia. 6. In Fig. 6 (top) contour lines of the event yield accumulated with the minimimum bias trigger are shown in the $p_{1}$ versus $p_{\| l}$ plane for the $\mathrm{Ca}+\mathrm{Ca}$ system. The peak at 
H.-A. GUSTAFSSON, et al.

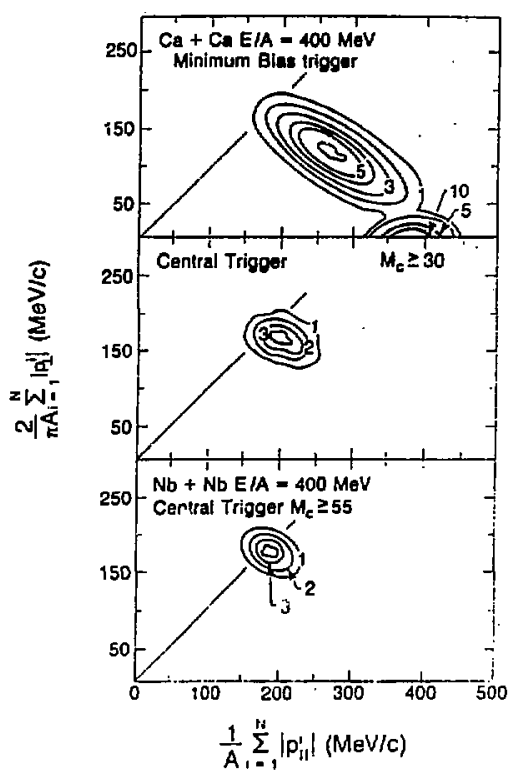

FIGURE 6 Contour plots of the average particle momentum components perpendicular and parallel to the beam axis for $\mathrm{Ca}+\mathrm{Ca}$ (top and center) and $\mathrm{Nb}$ $+\mathrm{Nb}$ (bottom) at $\mathrm{E} / \mathrm{A}=400 \mathrm{MeV}$. The diagonal line corresponds to isotropic events.

small $p_{\perp}$ but large $p_{\|}$corresponds to peripheral reactions and is dominated by projectile fragments. This contribution vanishes as the trigger is changed to a "central" one. Fig. 6 (center) shows central trigger events with a charged particle multiplicity larger than 30. The maximum of the yield is shifted towards the 
THE GSI-LBL PLASTIC BALL/WALL SPECTROMETER

diagonal but oniy a few events reach it, which corresponds to full stopping of the nuclei. In the lower part of Fig. 6 for the reaction of $400 \mathrm{MeV} /$. nucleon $\mathrm{Nb}+\mathrm{Nb}$ the central trigger events with charged particle niultiplicities $M_{c}$ larger than 55 almost fulfill the stopping and isotropy condition on the average.

\subsection{Collective Flow}

Direct evidence of the compression effects predicted by an equation of state would be collective flow of the nuclear matter upon re-expansion. Data from $4 \pi$ detectors like the Plastic Ball are ideally suited for studying the emission patterns and event shapes which might be able to reveal this effect and distinguish between predictions of cascade and hydrodynamical models.

The sphericity tensor

$$
F_{i j}=\sum_{v} p_{i}(v) p_{j}(v) w(v)
$$

is calculated from the momenta of all measured paricicles for each event. It is appropriate to choose the weight factor $w(v)$ in such a way that composite particles have the same weight per nucleon as the individual nuc?eons of the composite particle at the same velocity. For the Plastic Ball analyses the weight $w(v)=1 / 2 m(v)$ (kinetic energy flow) is used.

The sphericity tensor approximates the event shape by an ellipsoid whose orientation in space and whose aspect ratios can be calculated by diagonalization. The shapes predicted by hydrodynamical and intranuclear cascade calculations are quite different. The hydrodynamical model predicts prolate shapes along the beam 
H.-A. GUSTAFSSON, et al.

axis anly for grazing collisions but with decreasing impact parameter the flow angle increases, and reaches $90 \mathrm{deg}$. (with oblate shapes) for zero-impact-parameter events. Simple cascade calculations on the other hand predict zero flow angles at all impact parameters.

Approximately 50,000 Plastic Ball events accumulated with a minimum-bias trigger have been analyzed for the two systems $\mathrm{Ca}+\mathrm{Ca}$ and $\mathrm{Nb}+\mathrm{Nb}$ at $40 \mathrm{C}$ $\mathrm{MeV} /$ nucleon. ${ }^{6}$ The distribution of the flow angle $\theta$ (angle between the major axis of the flow ellipsoid and the beam axisl is shown in Fig. 7 for different multiplicity selections. A striking difference between the Ca and Nb data can be observed. For all but the highest multiplicity bins the distribution of the flow angles for the Ca data is peaked at 0 deg. For $\mathrm{Nb}$, however, there is a finite deflection angle increasing with increasing mutiplicity. The same analysis has been performed with $f i l$ tered events from a cascade cade calclitation (Fig. 7). For both systems studied the distributions are always peaked at $0 \mathrm{deg}$. It: is not so evident that the $C_{a}+C_{a}$ collision differs from iis simulation with the cascade model, whereas a new collective phenomenon defiritely appears in the larger-mass system which is not accounted for by the present cascade models.

In this analysis each event was parametrized by an ellipsoid, but it is of interest to study the shapes in more detail. The fact that finite flow angles are seen in the data indicates that in those events a reaction plane exists that is defined by the flow axis and the beam axis. ATl events can be rotated by the azimuthal angle determined by the flow analys is so that their 
THE GSI-LBL PLASTIC BALL/WALL SPECTROMETER

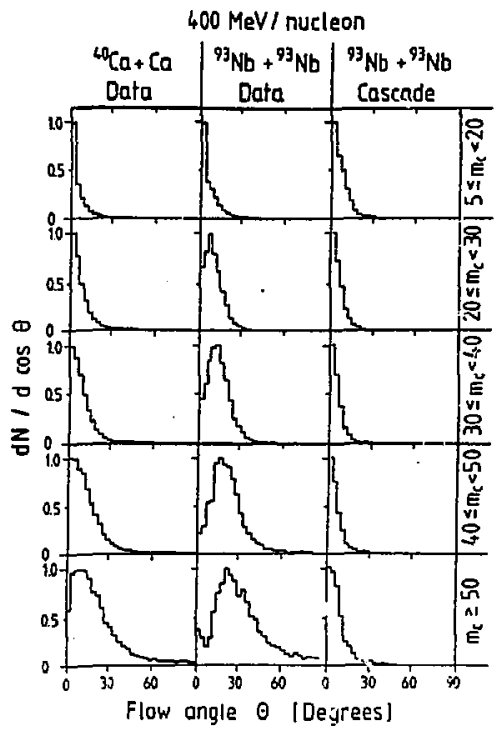

FIGURE 7 Frequency distributions of the flow angle $\theta$ for two sets of data and a cascade calculation for different multiplicity bins. For the case of Ca the multiplicities are half the indicated values.

individual reaction planes all fall into the $x-z$ plane, with the $z$ axis being the beam axis. For those rotated events the invariant cross section in the reaction plane $\left[d^{2} \sigma / d y d\left(p_{x} / m\right)\right]$ can be plotted, where $p_{x}$ is the projection of the perpendicular momentum into the reiction plane and $y$ is the center-of-mass rapidity. In addition, because of detector inefficiency near the 
H.-A. GUSTAFSSON, et al.

target rapidity, the graphs in Fig. $B$ have been

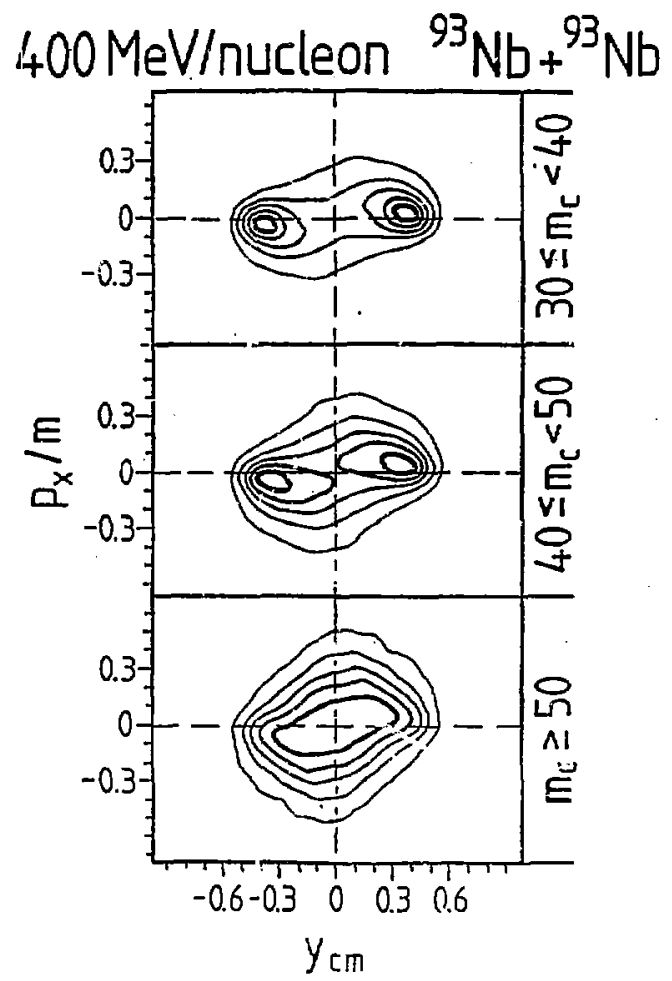

FIGURE 8 Contour plots (equal\}y-spaced linear contours without offset) of $P_{x} / m$ as a function of c.m. rapidity for multiplicities of 30-39, 40-49, and greater than 50 . 


\section{THE GSI-LBL PLASTIC BALL/WALL SPECTROMETER}

reflected about $90 \mathrm{deg}$. in the center of mass for didactic reasons.

The highest level contour, nea, projectile rapidity and just above the horizontal axis, results largely from the projectile remnants and indicates a definite bounceoff effect. The bounce-off process is a slowing down of the projectile fragments and a sidewards deflection in

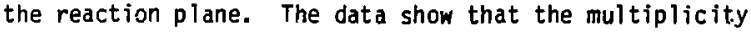
dependence of the crientation of the outer contour line's from the lower left to the upper right follows the trend indicated by the flow-angle distributions (Fig. 7). However, the position of the bounce-off peak from the projectile remnants changes only slightly with multiplicity. Thus one can coriclude that the strong sideward peaking seen in Fig. 7, which we will call side splash, is mainly due to the midrapidity particles. It should be noted that the bourice-off and side-splash effects appear to be in the same plane.

The hydrodynamical prediction of the rlow angle seems to be qualitatively in agreement with the measurement, but the prosent models do not predict two separate effects as seen in the data.

\subsection{Chemical Freeze-out Density}

One of the major goals in the study of composite particle production is to extract information about the size and density of the participant volume. The $d / p$ ratio determines the volume of the system at freezeout. To determine the density one needs the number of baryons in this volume. We define $N_{p}$ as the 
H.-A. GUSTAFSSON, et al.

participant baryon charge multiplicity. The participant baryon charge multiplicity, $N_{p}$, will be abbreviated to proton multiplicity.

The definition of dike is given by

$$
d_{\text {like }}=d+3 / 2(t+3 \mathrm{He})+3\left({ }^{4} \mathrm{He}\right)
$$

and the quartity $p_{\text {like }}$ is defined as

$$
p_{\text {like }}=p+d+t+2\left({ }^{3} \mathrm{He}+{ }^{4} \mathrm{He}\right)
$$

The functional form of the observed $\mathrm{d}_{1 \mathrm{ke}} / \mathrm{p}_{\text {like }}$ ratios $c$ an be understood in terms of the coalescence model. 7 We have used an improved version of the model which is a complete 6 dimensional phase space calculation relating the radi $i$ of the deuteron and the participant zone to the coordinate space, and the temperature of the interacting region to the momencum space. In this model the radius $r_{p}$ and the temperature $T$ of the interacting region as well as the deuteron radius $r_{d}$ are related to $d_{l i k e}$ and $p_{l i k e}$ through

$$
\begin{aligned}
& d_{\text {ike }} / p_{\text {like }}=6((A-Z) / Z)\left(1+2\left(r_{p} / r_{d}\right)^{2}\right)^{-3 / 2} N_{o} \times \\
& \left(1+2 m T r_{d}^{2} / 3\right)^{-3 / 2}
\end{aligned}
$$

where the factor for $(A-Z) / Z$ makes up for the difference between neutron and proton number and $m$ is the nucleon mass. The radius $r_{p}$ assuming a spherical source is parametrized as $r_{p}=r_{0}\left((A / Z) N_{p}\right)^{1 / 3}$, where $A / Z$ is the factor converting the participant baryon charge multiplicity to participant baryon multiplicity.

In the fits to the observed ratios, $r_{0}$ and $r_{d}$ were free parameters. The temperature $T$ was taken to be 


\section{THE GSI-LBL PLASTIC BALL/WALL SPECTROMETER}

the mean temperature obtained from Boltzmann fits $^{5}$ to the proton spectra at 90 deg. in the center-of-mass system. The fits to the experimental ratios are presented as solid curves in Fig. 9. The $r_{0}$ values

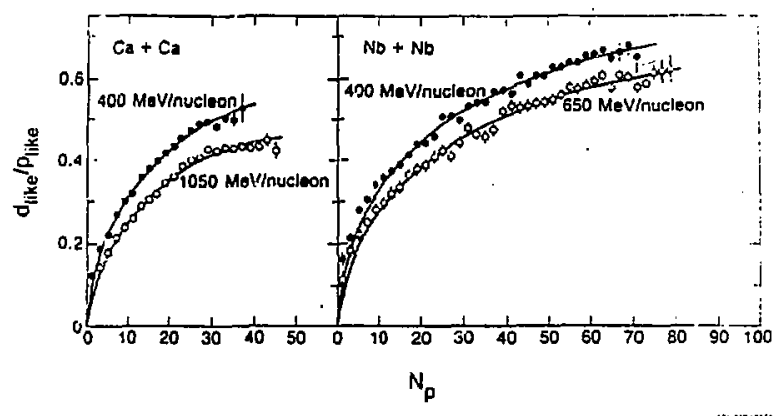

FIGJRE 9 dike/plike as a function of proton multiplicity $\left(\mathrm{N}_{\mathrm{p}}\right)$ for the two systems $\mathrm{Ca}+$ $\mathrm{Ca}$ at 400 and $1050 \mathrm{MeV} / \mathrm{nuc}$ leon and $\mathrm{Nb}+\mathrm{Nb}$ at 400 and $650 \mathrm{MeV} /$ nucleon. The curves shown are from $f$ its to eq. (6).

are the rms values for a Gaussian density distribution. To convert these values to equivalent sharp sphere radii the values have to be inultiplied by $\sqrt{5 / 3}$. The mean sharp sphere $r_{0}$ values obtained from the four cases studied correspond to freeze-out densities of 50 to 100 per cent times normal nuclear density.

\subsection{Thermal Freeze-out Density 8}

Proton-proton correlations wer? measured with the 
H.-A. GUSTAFSSON, et al.

Plastic Ball which has several features that are of significant value in this application: a large solid ang?e and rapid data-collection abllity, and the ability to detect most of the charged particles in each event, thus obtaining a direct measure of the participant multiplicity which is necessary for the determination of the freeze-out density.

Proton-proton correlations came into use for source-size measurements followng the correlation predictions of Koonin which, in addition to the secondorder interference effect due to quantum statistics, also include nuclear and Coulomb-induced final-state interactions between the two outgoing protons. At $\Delta p=$ 0 the correlation function is zero as a result of the Coulomb repulsion. A peak in the correlation function at $\Delta P=20 \mathrm{MeV} / \mathrm{C}$ is generated by the attractive nuclear force. The amplitude of this enhanced correlation is strongly dependent on the source-size parameter $r_{g}$ and increases as the source gets smaller. In the general form of Koonin's calculation the proton source density is treated as isotropic with a Gaussian distribution in space and time, and the proton momenta are assumed to be independent of position. The Gaussian spatial density, $p(r)=\exp \left[\left(-r / r_{g}\right)^{2}\right]$, may be thought of as representing the proton positions at the point of their last scattering. For our analysis we assume that the time parameter is zero, i.e., all the protons are emitted at the same time.

Radi i have been extracted by comparing measured correlation functions to theoretical predictions which depend on the source-size radius. In practice, the correlation function is obtained from the nata as a 
THE GSI-LBL PLASTIC BALL/WALL SPECTROMETER

function of $\Delta p$ (either one of the proton momenta transformed into the center of mass of the pair) by summing all p-p pairs, event by event, in bins $\left[N_{\text {true }}(\Delta p)\right]$ according to $\Delta p$, and dividing by $p-p$ pairs from different events $\left[N_{\text {mixed }}(\Delta p)\right]$ to give

$$
F(\Delta p)=\text { norm } \times N_{\text {true }}(\Delta p) / N_{\text {mixed }}(\Delta p) \quad .
$$

In forming the mixed-pair sums each proton was paired with each, oton in the preceding five events. It should ue pointer out that the Plastic Ball has a reduced efficie..cy for detecting pairs in the low-sp region since these pairs will tend to go botin into the same detector. However, the correlation function is well determined at $\Delta p=20 \mathrm{MeV}$ and above and thus gives a good measure of the radius. In Fig. 10 we show an example of a measured correlation function along with the distorted theoretical correlation function.

The extracted radii are shown as a function of proton multiplicity in Fig. 11. The error bars represent statistical errors only. For both the $\mathrm{Ca}+\mathrm{Ca}$ and $\mathrm{Nb}+\mathrm{Nb}$ systems the radius increases with multiplicity. The radii are shown with a fit using the function $r_{g}=r_{0}\left(N_{p} A / Z\right)^{1 / 3} /\left(\frac{5}{2}\right)^{1 / 2}$ where $r_{0}$ is a reduced radius (inversely proportional to the density). $N_{p}$ is the participant baryon charge multiplicity and $A / Z$ has been included to reflect the presence of neutrons. The extracted radii are the radius parameters for a Gaussian source distribution, and so by inclusion of $\left(\frac{5}{2}\right)^{1 / 2}$ in the above function $r_{0}$ becomes the radius parameter of an rms-equivalent sharp sphere. The value of $r_{0}$ extracted from the fits is $1.9 \mathrm{fm}$ for the two systems 
H. -A. GUSTAFSSON, et al.

$\mathrm{Ca}+\mathrm{Ca} 400 \mathrm{MeV} /$ nucleon

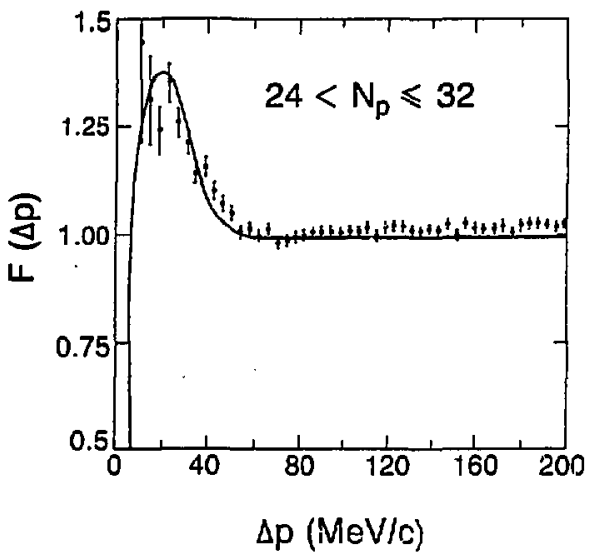

FIGURE 10 The measured proton-proton correlation function for $\mathrm{Ca}+\mathrm{Ca}$ with a proton multiplicity from 25 to 32 . The solid curve is a least-squares fit interpolation between distorted theoretical correlation functions for radii of 4 and $5 \mathrm{fm}$ which differ from each other by 25 per cent at the peak.

studied. Comparing this directly with $r_{0}=1.2 \mathrm{fm}$, the sharp-sphere $r_{0}$ for normal nuclei, yields a participant-region thermal freeze-out density about 25 per cent of normal nuclear density.

\section{CONCLUSION}

Results from the analyses of Plastic Ball data have been presented which show the various interrelations of the exclusive observables studied here. 
THE GSI-LBL PLASTIC BALL/WALL SPECTROMETER

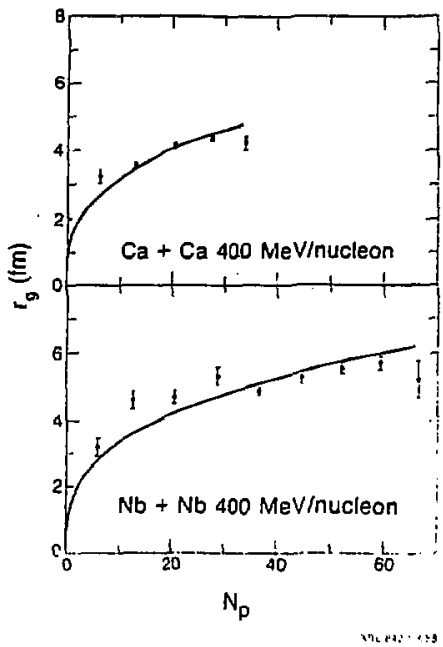

FIGURE 11 Extracted Gaussian source radii as a function of proton multiplicity $\left(N_{0}\right)$ for the two systems $\mathrm{Ca}+\mathrm{Ca}$ and $\mathrm{Nb}+\mathrm{Nb}$ at $400 \mathrm{MeVI}$ nucleon. The curves are fits to the results with the function $r_{g}=r_{0}\left(N_{\rho} A / Z\right)^{1 / 3} /\left(\frac{5}{2}\right)^{1 / 2}$.

From deuteron to proton ratios we are able to extract freeze-out densities for the chemical freeze-out stage occurring at earlier times in the expansion process than the thermal freeze-out stage which is accessible by two-particle correlation analysis. The two different collective effects - the bounce-off in the 


\section{H.-A. GUSTAFSSON, et àt.}

fragmentation region and the side-splash of the participants, which are prominent in the $\mathrm{Nb}+\mathrm{Nb}$ data, offer another piece of information that has to be included in a consistent description of high energy heavy ion interdetions. Up to now the hydrodynamical model including a full quantum statistical ca'culation for composite particle formation seems to be the most promising tool to describe the above mentioned features of the data and to finally deduce the nuclear equation of state.

\section{ACKMOHLEDGEMENTS}

Drs. A. M. Poskanzer and H. Lohner are thanked for their help in preparing this paper.

This work was supported by the Director, Office of Energy Research, Division of Nuclear Physics of the Office of High Energy and Nuclear Physics of the U.S. Department of Energy under Contract DE-ACn3-76SF00099.

\section{REFERENCES}

1. A. BADEN, H. H. GUTBROD, H. LÖH:ER, M. R. MAIER, A. M. POSKANZER, H. RIEDESEL, H. G. RITTER, H. SPIELER, A. WARWICK, F. WEIK and H. WIEMAN, NuCl. Instr. Meth. 203, 189 (1982)

2. A. SANDOVAL, H. H. GUTBROD, W. G. MEYER, A. M. POSKANZER, R. STOCK, J. GOSSET, HJ.-C. JOURDAIN, C. H. KING, G. KING, CH. LUKNER, NGUYER VAN SEN, G. D. WESTFALL and $K$. L. WOLF, Phys Rev. C21, 1321 (1980); W: G. MEYER, H. H. GUTBROO, CH. LUKNER and A. SANDOVAL, Phys. Rev, C22 179 (1980); A. I. WARWICK, A. BADEN, H. H. GUTBROD, M. R. MAIER, H. G. RITTER, H. STELZER, F. WEIK, H. WIEMAN, S. B. KAUFMAN, B. D. WILKINS, E. P. STE INBERG and $J$. PETER, Phys Rev. C27, 1083(1983)

3. R. STOCK, R. BOCK, R. BROCKMANN, J. W. HARRIS, A. SANDOVAL, H. STRÖBELE, K. L. WOLF, H. G. PUGH, L. 
THE GSI-LBL PLASTIC BALL/WALL SPECTROMETER

S. SCHROEDER, R. RENFORDT, A. OACAL and M. E. ORTIS, Phys. Rev. Lett. 49, 1236 (1982)

4. H. H. GUTBROD, M. R. MAIER, H. G. RITTER, A. I. WARWICK, F. WEIK, $H$, WIEMAN, and $K$. L. WOLF, IEEE Transactions on Nuclear Science, Vol. NS-28, No. 1 (February 198T)

5. H. -A. GUSTAFSSON, H. H. GUTBROD, B. KOLB, $H$. LÖHNER, B. LUDENIGT, A. M. POSKÁNZER, T. RENNER, H. RIEDESEL, H. RITTER, A. WARWICK and H. HIEMAN, Phys. Lett. 142B, 141 (1984)

6. H. A. GUSTAFSSON; H. H. GUTBROD, B. KOLB, H. LOHNER, B. LUDEWIGT, A. M. POSKANZER, T. RENNER, $H$. RIEOESEL, H. RITTER, A. WARWICK and H. WIEMAN, Phys. Rev. Lett. 53,1590 (1984)

7. H. H. GUTBROD, H. LÖHNER, A. Mo POSKANZER, T. RENIJER, H. RIEDESEL, H. RITTER, A. WARWICK, F. WEIK and H. WIEMAN, Phys. Lett. 127B, 317 (1983)

8. H.-A. GUSTAFSSON, H. H. BUTBROD, B. KOLB, H. LÖHNER, B. LLDEWIGT, A. M. POSKANZER, T. RENNER, $H$. RIEDESEL, H. RITTER, A. WARHICK, F. WEIK and $H$. WIEMAN, Phys. Rev. Lett. 53, 544 (1984)

9. 3. W. HARRIS and R. STOCK, LBL Report 17054 (1984) 
This report was done with support from the Department of Eneray. Any conclusions or opinjons expressed in this report represent solely those of the author(s) and not necessurily those of The Regents of the Univertity of California, the Lawrence Berkeley Laboratory or the Department of Energy.

Reference to a company or product name does not imply opproval or recommendation of the product by the University of Californie or the U.S. Department of Enersy to the exclusion of others that may be suitable 